\title{
B7-H6 is a new potential biomarker and therapeutic target of T-lymphoblastic lymphoma
}

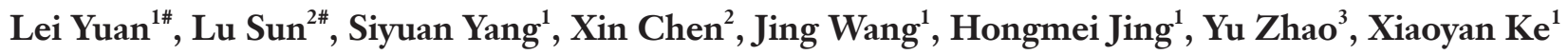 \\ ${ }^{1}$ Department of Hematology and Lymphoma Research Center, Peking University Third Hospital, Beijing, China; ${ }^{2}$ Department of Pathology, \\ Chinese PLA General Hospital, Beijing, China; ${ }^{3}$ Department of Hematology, Chinese PLA General Hospital, Beijing, China \\ Contributions: (I) Conception and design: X Ke; (II) Administrative support: L Yuan, J Wang; (III) Provision of study materials or patients: L Sun, \\ S Yang, H Jing, Y Zhao; (IV) Collection and assembly of data: L Yuan, L Sun; (V) Data analysis and interpretation: L Yuan, L Sun, X Chen; (VI) \\ Manuscript writing: All authors; (VII) Final approval of manuscript: All authors. \\ "These authors contributed equally to this work. \\ Correspondence to: Dr. Xiaoyan Ke. Department of Hematology and Lymphoma Research Center, Peking University Third Hospital, No. 49 North \\ Garden Road, Beijing 100191, China. Email: xiaoyank@yahoo.com; Dr. Yu Zhao. Department of Hematology, Chinese PLA General Hospital, No. \\ 28 Fuxing Road, Beijing 100853, China. Email: zhaoyu301@126.com.
}

\begin{abstract}
Background: B7-H6 is a novel co-stimulatory protein exclusively expressed on a variety of cancer cells and associated with poor prognosis. T-cell lymphoblastic lymphoma (T-LBL) is a highly aggressive hematological malignancy whose treatment requires reliable prognostic biomarkers and therapeutic targets. However, the rare nature and delayed progression of T-LBL have limited its clinical management.

Methods: The expression of B7-H6 was analyzed by immunohistochemistry (IHC) in 65 T-LBL samples; the association with the clinicopathological characteristics and prognosis was also investigated. B7-H6depleted Jurkat cells were also generated to investigate the effect of B7-H6 on cell proliferation, migration, and invasion. RNA sequencing was used to explore differentially expressed genes.

Results: B7-H6 was expressed in 61.5\% (40/65) of T-LBL patients; of note, 38.5\% (25/65) of patients showed membrane/cytoplasmic expression of B7-H6. Although the expression of B7-H6 varied across samples and did not correlate with patient survival, it was significantly associated with B symptoms, high ECOG scores (3 to 4), elevated serum lactate dehydrogenase level, and reduced complete remission at interim evaluation. B7-H6 underwent translocation into the nucleus of T-LBL cells, showing a specific nuclear localization sequence in the C-terminus. Moreover, the depletion of B7-H6 in Jurkat cells impaired cell proliferation, migration, and invasion. RNAseq showed the differential expression of RAG-1, which may be involved in the tumorigenesis of T-LBL.
\end{abstract}

Conclusions: B7-H6 may serve as a novel prognostic biomarker and therapeutic target of T-LBL.

Keywords: T-cell lymphoblastic lymphoma (T-LBL); B7-H6; RAG-1; prognostic biomarker; therapeutic target

Submitted Jul 14, 2020. Accepted for publication Nov 13, 2020.

doi: 10.21037/atm-20-5308

View this article at: http://dx.doi.org/10.21037/atm-20-5308

\section{Introduction}

T-cell lymphoblastic lymphoma/acute lymphoblastic leukemia (T-LBL/ALL) is a very aggressive subtype of non-Hodgkin lymphoma (NHL) most frequently detected in late childhood and young adulthood (1). T-LBL comprises $15-25 \%$ of all ALL cases and $2 \%$ of adult
NHL cases, especially in males $(2,3)$. T-LBL is associated with very poor prognoses, with a 5 -year survival rate of $30-50 \%$, despite the use of treatment approaches such as intensified chemotherapy and hematopoietic stem cell transplantation (4). Recent reports have identified a number of prognostic factors and therapeutic targets in the context of T-LBL. The expression of CD1a and the lack 
of the expression of CD13 are associated with improved survival in patients with T-LBL (5). Importantly, complex cytogenetic abnormalities were reported to further lower the 5 -year survival of T-LBL patients to $19 \%$ (5). Patients are categorized into "low-risk" and "high-risk" prognosis groups based on mutations in NOTCH1 and/or FBXW7 (N/F), K-RAS, and PTEN (6-8); of note, although ALL and T-LBL are currently considered the same disease with different clinical manifestations, these observations are pertinent for ALL, but not for T-LBL. However, the above-mentioned classifications are not generally used in the context of daily clinical practice. Moreover, there are limited options currently available for the treatment of T-LBL $(9,10)$. Thus, it is imperative to identify novel targets and biomarkers to better predict and improve the prognosis of T-LBL.

B7-H6 is a novel co-stimulatory protein exclusively expressed on a variety of cancer cells; B7-H6 is a ligand of NKp30, an activating receptor on natural killer (NK) cells $(11,12)$. The non-immunological and immunological roles of B7-H6 are currently under investigation (13). The majority of the previous studies have shown that B7H6 is a poor prognostic factor for various cancers (14-25), excluding gastric carcinoma (26). On the other hand, NKT cells expressing NKp30 in patients with melanoma are associated with improved prognosis $(27,28)$. In fact, clinical studies have suggested the use of antibody- or NKp30based chimeric antigen receptor $\mathrm{T}(\mathrm{CAR}-\mathrm{T})$ cells for the determination of the expression of B7-H6 in tumors (29-31). Of note the expression of $\mathrm{B} 7-\mathrm{H} 6$ in the membrane of tumor cells was reported to increase upon inhibiting metalloprotease (MMP)-mediated shedding or chemotherapy, enhancing the efficacy of NK cell-based cancer therapies $(32,33)$. In contrast, histone deacetylase inhibitors were reported to impair NK cell-mediated tumor cell recognition via the downregulation of B7-H6 (34).

We have previously shown that $\mathrm{B} 7-\mathrm{H} 6$ promotes cancer cell migration and aggressiveness and reduces apoptosis via the STAT3 pathway in B cell NHL (21). Of note, B7-H6 is expressed on the Jurkat and MOLT-4 cell lines, derived from patients with T cell ALL. However, the effect of B7-H6 on T-LBL/ALL is not yet fully understood. This study aimed at the determination of the expression and location of B7-H6 in 40/65 paraffin-embedded tissues from individuals with T-LBL. Furthermore, we have explored the correlation between B7-H6 levels and the clinicopathological characteristics, response to therapy, and prognosis of patients using survival analysis. Importantly, this study is in accordance with the REMARK reporting checklist (available at http://dx.doi.org/10.21037/atm-205308).

\section{Methods}

\section{Clinical data}

We used specimens from 89 patients with T-LBL admitted to the Department of Hematology from the Peking University Third Hospital, and to the PLA General Hospital between 2008 and 2019. The clinical characteristics of all patients were collected from the relevant electronic medical databases and listed in Table 1. We excluded 24 samples due to incomplete data. All of the patients included were administered chemotherapy agents recommended for the treatment of ALL. Patients underwent interim response evaluation via computed tomography scan prior to their third cycle of chemotherapy. Twenty normal tissues from 18 lymph nodes (LNs) and 2 thymuses were used as negative controls. Of note, patients with a poor clinical condition upon diagnosis who were administered 1-2 cycles of cyclophosphamide, doxorubicin, vincristine, and prednisone (CHOP) before the adoption of intensified ALL or ALL-like chemotherapy were also enrolled in this study. All pathological data were reviewed independently by two senior pathologists (LS and XC). Follow-up data were available for all the patients up to January 2020 with a follow-up time ranging between 6 and 132 months.

The study was conducted in accordance with the Declaration of Helsinki (as revised in 2013). The study was approved by the Institutional Review Board of Peking University Third Hospital (No. 2016060). Due to the study's retrospective nature, written informed consent was waived.

\section{Immunobistochemistry (IHC)}

IHC was performed according to the instructions provided below. We used paraffin-embedded 3- $\mu$ m thick lymph node sections from biopsies. All the sections were de-waxed using xylene and rehydrated with ethanol and water. Citrate buffer ( $\mathrm{pH}$ 6.0) was used to retrieve antigens at $95^{\circ} \mathrm{C}$ for $2 \mathrm{~min}$. We used hydrogen peroxide (3\%) to block the activity of endogenous peroxidase for $10 \mathrm{~min}$ followed by rinsing with Tris-buffered saline. Subsequently, sections were incubated at room temperature with primary 
Table 1 Characteristics of the 65 T-LBL patients enrolled in this study.

\begin{tabular}{|c|c|c|c|c|}
\hline Characteristics & Cases $(n=65)$ & \multicolumn{3}{|c|}{$\mathrm{B} 7-\mathrm{H} 6$ protein expression } \\
\hline Gender & & & & 0.36 \\
\hline Male & 53 & 19 & 34 & \\
\hline Female & 12 & 6 & 6 & \\
\hline$\leq 30$ & 45 & 15 & 30 & \\
\hline$>30$ & 20 & 10 & 10 & \\
\hline Ann Abor stage ${ }^{\dagger}$ & & & & 0.79 \\
\hline III & 6 & 2 & 4 & \\
\hline$\leq 500$ & 42 & 20 & 22 & \\
\hline$>500$ & 23 & 5 & 18 & \\
\hline ECOG score & & & & 0.02 \\
\hline $0-2$ & 50 & 23 & 27 & \\
\hline $3-4$ & 15 & 2 & 13 & \\
\hline IPI score ${ }^{\ddagger}$ & & & & 0.69 \\
\hline $0-2$ & 37 & 15 & 22 & \\
\hline 3 & 28 & 10 & 18 & \\
\hline Yes & 44 & 18 & 26 & \\
\hline No & 21 & 7 & 14 & \\
\hline Mediastinum mass & & & & 0.97 \\
\hline Yes & 47 & 18 & 29 & \\
\hline No & 18 & 7 & 11 & \\
\hline Interim response ${ }^{\S}$ & & & & 0.02 \\
\hline $\mathrm{CR}$ & 37 & 18 & 19 & \\
\hline Not CR & 19 & 3 & 16 & \\
\hline
\end{tabular}

${ }^{\dagger}$, no patient with Ann Abor stage of I-II in the entire cohort; ${ }^{\ddagger}$, only 1 patient with IPI of $\leq 1$ in the whole cohort; ${ }^{\S}, 9$ patients failed to complete interim evaluation were excluded (4 in B7-H6 negative and 5 in positive group, respectively). LDH, lactate dehydrogenase; IPI, international prognostic index; BM, bone marrow; $\mathrm{CR}$, complete remission. 
Table 2 The sequence of PCR primer pairs and siRNA, shRNA targeting B7H6

\begin{tabular}{lcc}
\hline Name & Sequences & Annealing temperature, ${ }^{\circ}$ C/cycles \\
\hline Primer: B7-H6-Q-F & CTTTACCCTGACTGCTGC & $60 / 40$ \\
Primer: B7-H6-Q-R & ATATGAGGTGCTCTTTCTTC & $60 / 40$ \\
Primer: RAG1-Q-F & TTCTGCCCCAGATGAAATTC & $60 / 40$ \\
Primer: RAG1-Q-R & TGACCATCAGCCTTGTCCAG & $60 / 40$ \\
Primer: GAPDH-Q-F & TCAAGGCTGAGAACGGGAAG & $60 / 40$ \\
Primer: GAPDH-Q-R & TCGCCCCACTTGATTTTGGA & $60 / 40$ \\
siRNA-B7H6 & CATCTTCAGCCTATACTCCTCTCAA \\
shRNA-B7H6: top strand & GATCCGCATCTTCAGCCTATACTCCTCTCAATTCAAGAGATTGAG \\
shRNA-B7H6: bottom strand & AGGAGTATAGGCTGAAGATGTTTTTG & \\
& TGAGAGGAGTATAGGCTGAAGATGCG & \\
\hline
\end{tabular}

rabbit anti-human B7-H6 polyclonal antibody (Abcam, Cambridge, MA, catalog number 121794; diluted 1:400 in antibody diluent) for $60 \mathrm{~min}$. After a new rinsing step with Tris-buffered saline, sections were incubated with secondary horseradish peroxidase-labeled IgG anti-rabbit for $40 \mathrm{~min}$.

The IHC data from previous reports have shown the membrane and cytoplasmic localization of B7-H6 protein in tumor cells $(19,20,22,26,35)$. Thus, we measured both the percentage and staining intensity [negative (-), weakly positive $(+)$, moderately positive (++), and strongly positive $(+++)]$ of positively stained cells. Tissue sections were observed using an Olympus BX53 microscope (Olympus Corporation, Japan) at $\times 400$ magnification. Using at least five randomly selected fields per section irrespective of the intensity, the percentage and intensity of $\mathrm{B} 7-\mathrm{H} 6$ positive cells were calculated independently by two experienced hematopathologists (LS and XC). In brief, unequivocal staining in $>10 \%$ of tumor cells on the membrane, cytoplasm, and/or nucleus was defined as positive; we used a positive $\mathrm{B} 7-\mathrm{H} 6$ staining threshold of $10 \%$ owing to the limited number of cases enrolled in this study and for convenience in the context of the daily clinical practice, regardless of the IHC staining intensity.

\section{Cell culture}

The T-LBL cell line Jurkat (Jurkat-wt, clone E6-1, ATCC, TIB-152), the B7-H6 knockdown derivative (Jurkatsh-B7H6), and control cells transfected with the mock lentiviral vector (Jurkat-sh-Luc) were cultured at $37^{\circ} \mathrm{C}$ and $5 \% \mathrm{CO}_{2}$ in RPMI-1640 (Gibco) with $10 \%$ heatinactivated fetal bovine serum (FBS) and $1 \%$ penicillin/ streptomycin as described previously (36). The Jurkat cell line was purchased from ATCC and maintained in our laboratory. Human embryonic kidney 293T (HEK-293T) cells (Invitrogen, Carlsbad, CA USA) were cultured in high glucose Dulbecco's modified Eagle medium (DMEM) supplemented with $10 \%$ FBS (Thermo Fisher Scientific, Waltham, MA, USA) at $37^{\circ} \mathrm{C}$ and $5 \% \mathrm{CO}_{2}$.

\section{$R N A$ interference and lentiviral infection}

A lentiviral vector expressing the B 7-H6specific small hairpin RNA (shRNA; HanBio Cop. Shanghai, China) was used to deplete cells of B7-H6. The target sequence of the siRNA was 5'-CATCTTCAGCCTATACTCCTCTCAA-3' (Table 2). To generate the recombinant lentiviruses, HEK-293T cells were seeded into $15-\mathrm{cm}$ cell culture dishes and cultured for $24 \mathrm{~h}$ prior to transfection to allow the obtention of $70-80 \%$ confluent cultures. The two packaging plasmids pSPAX2 and pMD2G were co-transfected into HEK-293T cells using the Lipofiter ${ }^{\mathrm{TM}}$ Liposomal Transfection Reagent according to the manufacturer's instructions (HanBio Cop. Shanghai, China). The cell culture medium was replaced with fresh complete medium $6 \mathrm{~h}$ after transfection. Fortyeight to $72 \mathrm{~h}$ after transfection, the culture medium was collected for the isolation of the lentiviruses.

The titration of lentiviruses was performed using HEK-293T cells previously seeded into 96-well plates (1× 
$10^{5}$ cells $/ \mathrm{mL}$ in a volume of $100 \mu \mathrm{L} /$ well) and cultured for $24 \mathrm{~h}$. Cells were then transduced with 6 dilutions of the vectors, cultured for $72 \mathrm{~h}$. Puromycin-resistant cells were then quantified using fluorescence microscopy. The titers were calculated based on the following formula:

Lentivirus titer $=$ cell number $\times$ (percentage of fluorescent positive cells/100) $\times$ Vector dilution factor $\times$ $\mathrm{MOI} \times 10^{3} \mathrm{TU} / \mathrm{mL}$

The Jurkat cells were transduced using polybrene (Sigma Aldrich, St. Louis, MO, USA) following the manufacturer's instructions. A total of 300,000 cells were seeded before transfection into 24-well plates and then infected in the presence of $5 \mu \mathrm{g} / \mathrm{mL}$ polybrene (Sigma Aldrich). Puromycin $(2 \mu \mathrm{g} / \mathrm{mL})$ was used as the selection agent. Knockdown of B7-H6 was confirmed by qRT-PCR and western blotting, $72 \mathrm{~h}$ after transfection.

\section{Quantitative reverse transcription-polymerase chain reaction ( $q R T-P C R)$}

Total RNA was extracted using TRIzol (Life Technologies, Carlsbad, CA, USA) in accordance with the manufacturer's protocol. cDNA was synthesized using the ReverTra Ace qPCR RT Kit (FSQ-101, Toyobo, Osaka, Japan) and a Thermal Cycler (Bio-Rad Laboratories, Hercules, CA, USA). Quantitative PCRs were performed using the LightCycler ${ }^{\circledR} 96$ System (Roche Diagnostics, Indianapolis, IN, USA) and SYBR Green according to the manufacturer's instructions. GAPDH was used as an internal standard gene, and the relative gene expression was calculated using the $2^{-\triangle \Delta C T}$ method. The cycling conditions for and the sequences of primers used in this study are listed in Table 2.

\section{Cell proliferation, migration, and invasion assays}

For the evaluation of cell proliferation, we seeded 3,000 cells/well in triplicates in 96-well pre-coated culture plates. Twenty-four, 48, 72, and $96 \mathrm{~h}$ after transfection, $10 \mu \mathrm{L}$ of Cell Counting Kit-8 reagent (CCK-8, Dojindo, Kumamoto, Japan) was added to the medium and incubated for $3 \mathrm{~h}$ at $37^{\circ} \mathrm{C}$. The absorbance at $450 \mathrm{~nm}$ was measured using a Multiskan GO microplate spectrophotometer (Thermo Fisher Scientific, Waltham, MA, USA). This experiment was repeated 3 times.

Cell migration was evaluated using Transwell chambers (24-well insert; Corning, Inc., Corning, NY, USA). A total of 50,000 Jurkat, Jurkat-shLuc, and Jurkat-shRNAB7H6 cells suspended in RPMI-1640 containing 1\% FBS and seeded into the upper chamber of the insert $48 \mathrm{~h}$ after transfection. RPMI-1640 containing 15\% FBS was added to the lower chamber to serve as a chemoattractant. Cells were allowed to migrate for $24 \mathrm{~h}$. The cells on the upper surface of the membrane were collected using a cotton bud along with the migrating cells in the lower chamber. These cells were subjected to light microscopy and photographed (COIC, Chongqing, China). In some experiments, we added Matrigel (BD Bioscience, Corning, NY, USA) to evaluate cell invasion. These experiments were repeated three times.

\section{Sequencing}

Sequencing libraries were generated using the NEBNext ${ }^{\circledR}$ Ultra $^{\mathrm{TM}}$ RNA Library Prep Kit for Illumina ${ }^{\circledR}$ (NEB, Ipswich, MA, USA) following the recommendations of the manufacturer; index codes were added to attribute sequences to each sample. We performed PCR using the Phusion High-Fidelity DNA polymerase, universal PCR primers, and Index $(X)$ Primer. PCR products were purified (AMPure XP system), and the quality of the library was assessed using the Agilent Bioanalyzer 2100. Differential gene expression was analyzed using the DESeq $\mathrm{R}$ package (1.20.0). $\mathrm{P}$ values were adjusted using the Benjamini and Hochberg's approach to control the false discovery rates. A corrected $\mathrm{P}$ value of 0.005 and a $\log 2$ (Fold change) of 1 were set as the threshold for differential gene expression.

\section{Analysis of the nuclear binding domain}

We searched the BioGRID platform to identify the possible interactions between B7-H6 and other proteins $(37,38)$. We selected XPO-5, part of the karyopherin $\beta$ family of transport factors, that induces the nucleocytoplasmic shuttling of proteins (39). We further predicted nuclear localization sequence (NLS) recognition by Kap $\beta 2$ :NLSs using the following three rules: (I) NLS is structurally located in the substrate; (II) NLS is positively charged; (III) consensus sequences are associated with NLS (40). We used a central hydrophobic or basic motif followed by a C-terminal $\mathrm{R} / \mathrm{H} / \mathrm{KX}(2-5) \mathrm{PY}$ consensus sequence as the control.

\section{Statistical analysis}

Statistical analyses were performed using Stata, version 14.0 (Stata, Houston, TX, USA) and the R software (R 
version 3.63, www.R-project.org). The GraphPad prism 8.0 (GraphPad Software, La Jolla, CA, USA) was used to obtain the data graphical representations. The $\chi^{2}$ test was used to analyze the correlation between the expression of B7-H6 and the patients' clinicopathological parameters. KaplanMeier plots were used to represent the overall survival of the patient cohort. Differences in the survival were assessed using the log-rank test. $\mathrm{P}<0.05$ was considered statistically significant.

\section{Results}

\section{B7-H6 is overexpressed in T-LBL tissues}

B7-H6 was overexpressed in T-LBL and detected in 61.5\% (40/65) of the T-LBL patient samples (Figure 1, Table 1). Consistent with its function as a structural membrane glycoprotein, B7-H6 predominantly localized to the membrane and cytoplasm of cells in the tissues collected from patients with T-LBL. We also observed the expression of B7-H6 in the cytoplasm of plasma cells infiltrating the T-LBL tumor (4 cases, figures not shown). Furthermore, with respect to the 20 normal tissues, seven were positive for $\mathrm{B} 7-\mathrm{H} 6(\mathrm{P}=0.037)$. Of note, $\mathrm{B} 7-\mathrm{H} 6$ was expressed in the subcortical regions of the normal $\mathrm{LNs}(\mathrm{n}=7)$, whereas the remaining regions were negative. In addition, B7-H6 was only expressed in the nucleus of cells (most probably $\mathrm{T}$ cells) with weak intensity in that region (Figure 1C,D). We were unable to distinguish between the expression of B7-H6 in the cytoplasm or in the membrane of cells from T-LBL samples since they had very limited cytoplasm. Therefore, we combined the two patterns of cytoplasmic expression of B7-H6 (Figure 1E). Among the 40 positive cases, $57.5 \%(23 / 40)$ showed positive staining for B7-H6 in the cytoplasm (Figure 1E). B7-H6 staining was also detected exclusively in the nucleus (Figure $1 F$ ) and in both the cytoplasm and the nucleus in $37.5 \%(15 / 40)$ and $5.0 \%(2 / 40)$ of the cases, respectively (Figure 1B).

The intensity of B7-H6 expression in T-LBL was weak (+; $16 / 40 ; 40.0 \%$ of the cases, Figure 17$)$ to moderately positive $(++; 21 / 40 ; 52.5 \%$ of the cases, Figure $1 \mathrm{~K})$; only $7.5 \%$ (3/40) of the cases were strongly positive (+++, Figure $1 L)$. Although statistically insignificant, we observed a positive correlation between the study population and the intensity of B7-H6-positive tumor cells (Figure 1A). However, this phenomenon was neither observed for the frequency of B7H6-positive T-LBL cells, nor for the localization of B7-H6 (Figure 1B).

\section{Correlation between the expression B7-H6 and the clinicopathological features of T-LBL patients}

Table 1 summarizes the correlation between the expression of B7-H6 and the T-LBL-related clinical features. The expression of $\mathrm{B} 7-\mathrm{H} 6$ was associated with tumor samples with an ECOG score $\geq 3(\mathrm{P}=0.02)$, lactate dehydrogenase levels $\geq 500 \mathrm{U} / \mathrm{L}(\mathrm{P}=0.04)$, and $\mathrm{B}$ symptoms (fever, weight loss, and night sweats) $(\mathrm{P}=0.01)$. However, other characteristics such as the Ann Abor stage, international prognostic index, and mediastinum mass did not correlate significantly with the expression of B7-H6. Since the Ki67 proliferation index was $>90 \%$ in almost all cases, no correlation analysis was performed. Overall, our data suggested that the expression of B7-H6 increased tumor burden. Thus, we further explored the potential clinical implications of the expression of B7-H6. We divided the cohort into 2 groups (B7-H6-positive and B7-H6-negative) based on the expression of B7-H6. Probably owing to the interference caused by response adjusted therapy, the overall survival was not significantly different between the groups (Figure 1M). Thus, we made the appropriate comparisons in the context of response to chemotherapy (Table 1). Interestingly, the expression of $\mathrm{B} 7-\mathrm{H} 6$ was not dependent on the sex or age of the patients. Of note, data were not available on the interim response evaluation for nine patients [B7-H6 positive group $(\mathrm{n}=5)$, negative group $(n=4)]$ since consent was not provided by the patients at this stage. Using interim response evaluation, we found a significant difference in complete remission between the patients in the two groups; more patients in the B7H6 negative group achieved complete remission $(87.5 \%$, $14 / 16$ cases) as compared to patients expressing B7-H6 (53.3\%, 16/30 cases; $\mathrm{P}=0.02)$. These results suggest that T-LBL patients expressing B7-H6 require intensified chemotherapy and targeted therapy.

\section{B7-H6 knockdown suppresses cell proliferation, migration, and invasion}

To further explore the role of $\mathrm{B} 7-\mathrm{H} 6$ in the tumorigenesis of T-LBL/ALL, we downregulated the expression of B7H6 in a highly invasive T-LBL/ALL cell line (Jurkat cells) using lentiviruses encoding a B7-H6 shRNA, and evaluated cell proliferation, migration, and invasion. The knockdown efficiency of B7-H6 was $69 \%$ in Jurkat-sh-B7H6 cells, as per qRT-PCR results with western blotting (Figure $2 A$ ). Importantly, we observed a significant reduction in the 
A

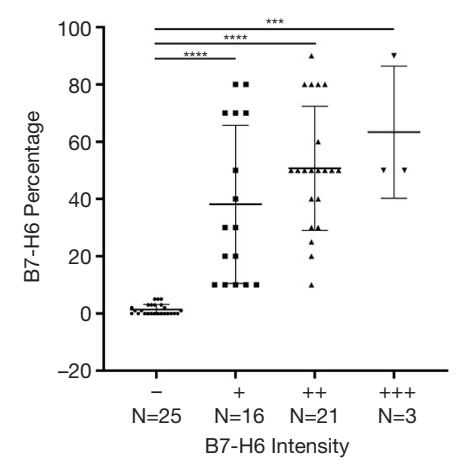

B

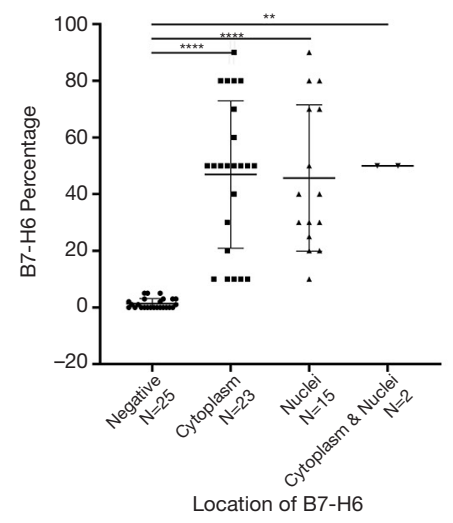

M

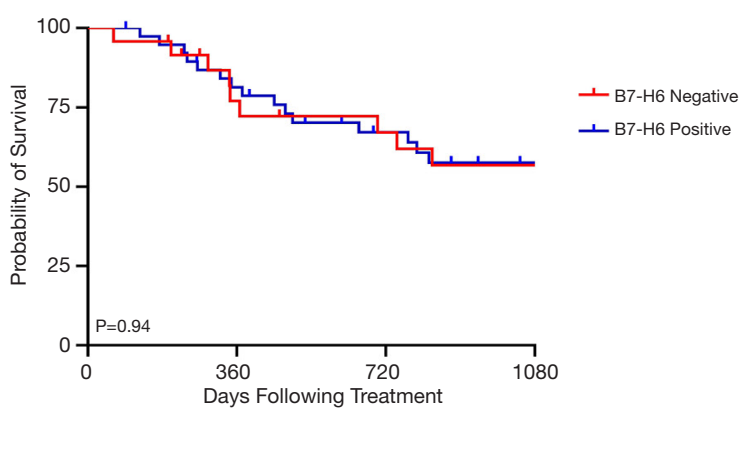

negative LN

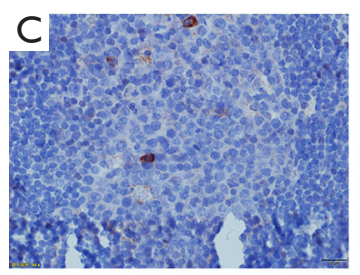

positive LN

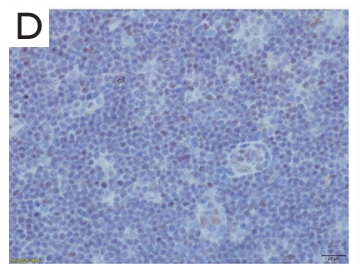

cytoplasm positive

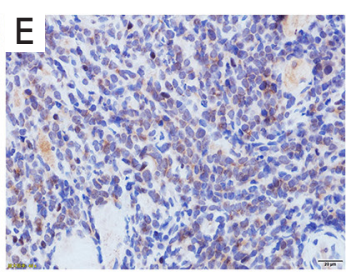

$20 \%$ positive

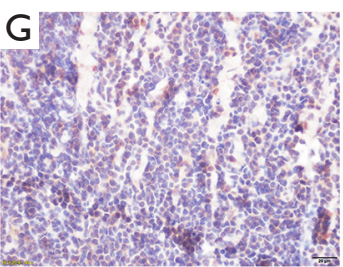

$50 \%$ positive

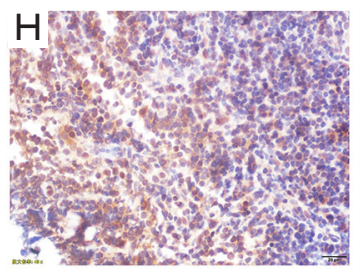

$80 \%$ positive

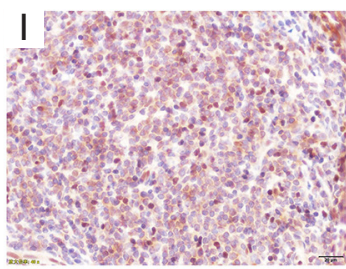

weak

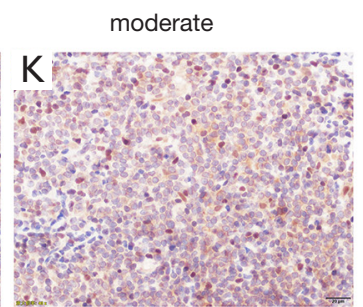

nuclei positive

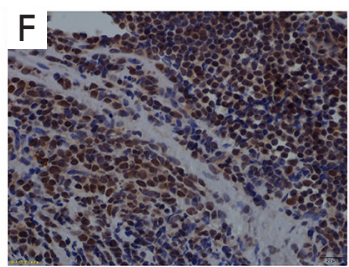

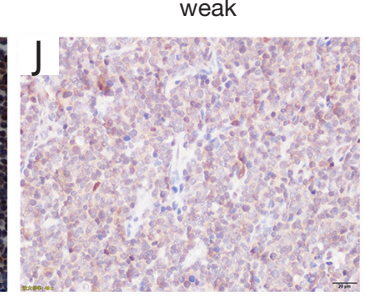

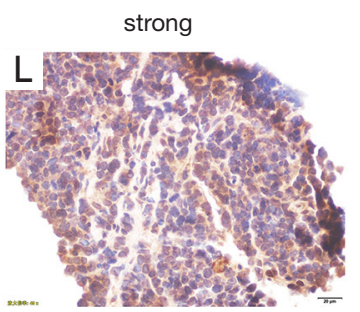

Figure 1 B7-H6 expression in human T-LBL tissues. (A) A trend that the proportion of positive cells increased with the staining intensity of B7-H6 in human T-LBL tissues; (B) the proportion of B7-H6 positive cells was similar nevertheless the location. B7-H6 expression on normal LNs (C: negative, D: positive; $\times 400)$. B7-H6 expressed on the membrane and/or in the cytoplasm (E, $\times 400)$, and in the nuclei (F, $\times 400)$. Representative T-LBL cases immunostained for B7-H6 with various levels of intensity and frequency. B7-H6 frequency (G: 20\%, H: $50 \%$, I: $80 \% ; \times 400$ ) or intensity (J: weak, K: moderate, L: strong; $\times 400)$. The Kaplan-Meier curve of overall survival, T-LBL patients with negative B7-H6 expression was similar with that of the patients with positive B7-H6 expression (K). The statistical significance of the comparisons is indicated as follows: ns, not significant; ${ }^{* *} \mathrm{P} \leq 0.01,{ }^{* * *} \mathrm{P} \leq 0.001,{ }^{* * * *} \mathrm{P} \leq 0.0001$. T-LBL, T-cell lymphoblastic lymphoma; LNs, lymph nodes.

proliferation, migration, and invasion of Jurkat-shB7H6 cells as compared to that of Jurkat-sh-Luc cells (Figure 2B,C,D).

\section{B7-H6 expression correlates with T-LBL aggressiveness and unfavorable prognosis}

In line with previous reports, RNAseq did not show a correlation between the expression of $\mathrm{B} 7-\mathrm{H} 6$ and T-LBLrelated biomolecules in Jurkat-sh-Luc and Jurkat-shB7H6 cells (Table 3) $(7,8,41)$. However, our RNAseq data showed that RAG-1 was differentially expressed ( $\log 2$ fold change, $-1.792676147 ; \mathrm{P}=2.96 \mathrm{E}-185)$ which was validated by qRT-PCR (Figure 3). RAG-1 drives the development of B-ALL and T-ALL. ADAMTS-17 was also differentially expressed to a low extent in Jurkat cells 
A
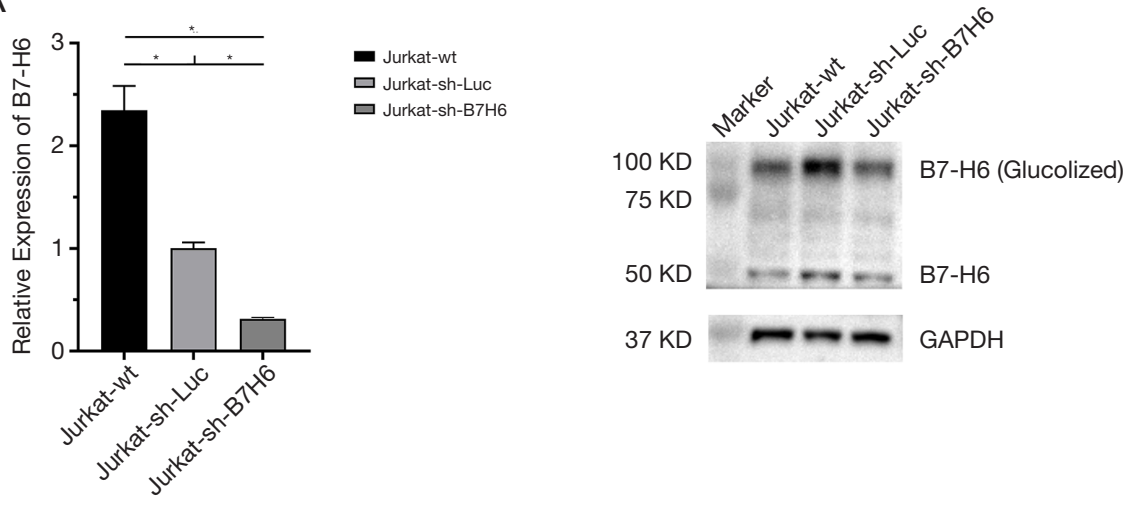

B

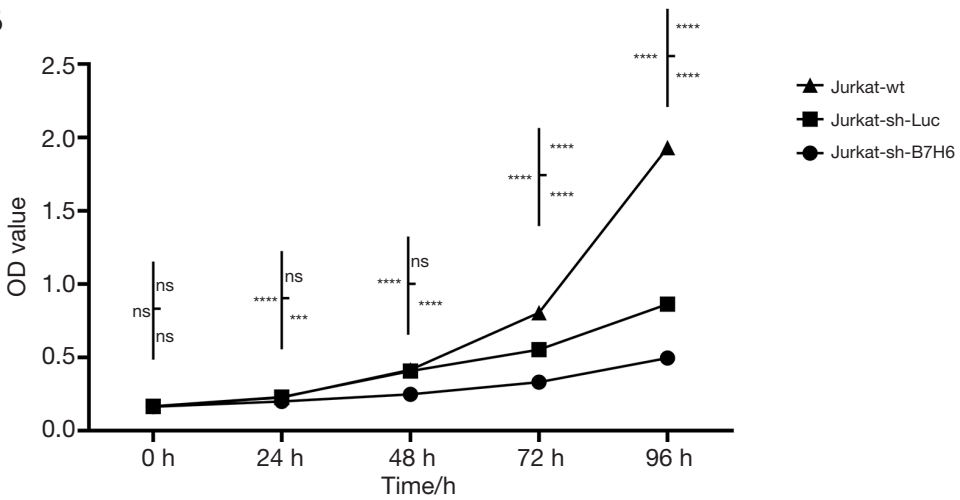

C

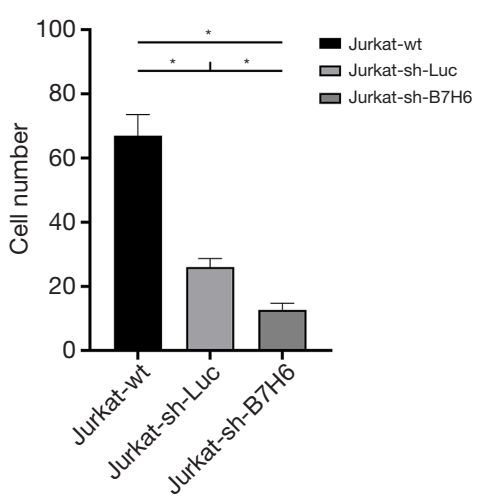

D

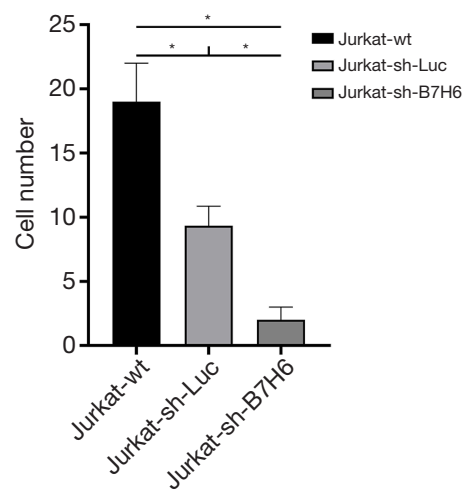

Figure 2 Establishment of stable knockdown of B7-H6 expression in T-lymphoblastic leukemia cell line Jurkat. The knockdown efficiency was confirmed by real-time RT-PCR and western blotting analysis, and the data showed that the expression level of B7-H6 was significantly decreased in the Jurkat-sh-B7H6 compared with Jurkat-sh-Luc group cells (A). The proliferation (B), migration, (C) and invasion (D) ability of the Jurkat cell line after knockdown B7-H6 expression in the Jurkat-sh-B7H6 group was examined compared with the Jurkat-sh-Luc group cells. The statistical significance of the comparisons is indicated as follows: ns, not significant; ${ }^{*} \mathrm{P} \leq 0.05,{ }^{* * *} \mathrm{P} \leq 0.001,{ }^{* * * *} \mathrm{P} \leq 0.0001$.

( $\log 2$ fold change, $-0.686892977 ; \mathrm{P}=1.25167 \mathrm{E}-28)$ and may regulate their potential for invasion. Although the function of ADAMTS-17 has not yet been fully understood, a study reported that it increases tumor aggressiveness upon secretion into the extracellular matrix (42). Taken together, these observations suggest that the levels of 
Table 3 Dataset for oncogenic signature

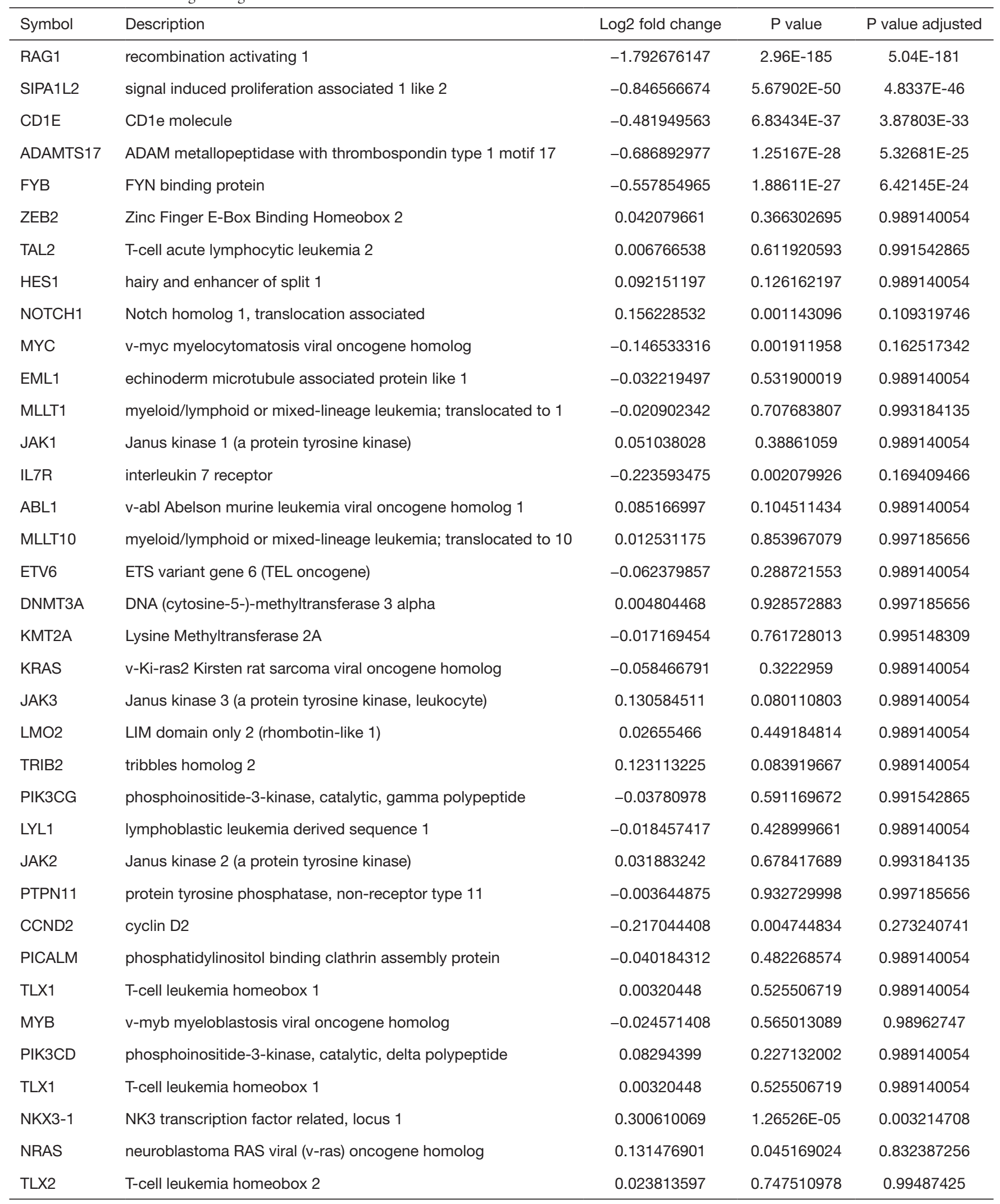




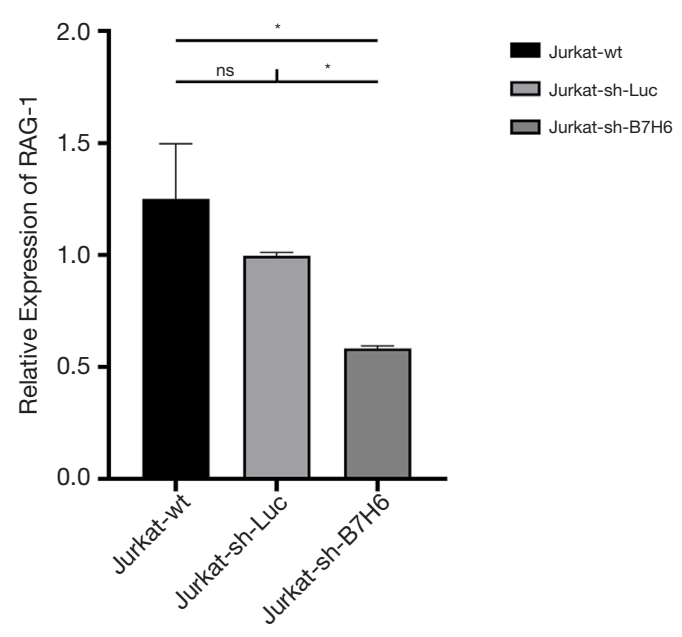

Figure 3 RAG-1 expression validated by real-time RT-PCR. The mRNA expression level of RAG-1 was significantly decreased in the Jurkat-sh-B7H6 compared with Jurkat-sh-Luc group cells (A). The statistical significance of the comparisons is indicated as follows: ns, not significant; ${ }^{*} \mathrm{P} \leq 0.05$. RT-PCR, reverse transcription-polymerase chain reaction.

B7-H6 correlate with T-LBL tumor aggressiveness and poor prognosis. Therefore, B7-H6 may serve as a novel prognostic biomarker of T-LBL.

\section{Discussion}

In this study, we investigated the expression of B7-H6 in T-LBL; moreover, we determined the correlation between the expression of $\mathrm{B} 7-\mathrm{H} 6$ and factors related to the poor prognosis of T-LBL. Importantly, we demonstrated that the downregulation of B7-H6 in Jurkat cells was associated with a decrease in their tumorigenic properties. To the best of our knowledge, this is the first report on the nuclear translocation of B7-H6. B7-H6 showed a nuclear localization pattern in cells of tissues from patients with T-LBL. This could be attributed to the presence of an NLS. Finally, this study showed that B7-H6 is a potential biomarker for the poor prognosis of T-LBL and may be a potential therapeutic target.

B7-H6 correlated with poor prognostic factors of T-LBL, such as elevated levels of lactate dehydrogenase, ECOG, and B symptoms. Previously, B7-H6 was associated with reduced survival after surgery in the context of several solid tumors (14-24,35). However, in this study, the expression of T-LBL was not predictive of reduced overall or progression-free survival. Since T-LBL is a highly aggressive subtype of lymphoma with extremely poor prognosis, the treatment regimen is restricted to multi-drug intensified chemotherapy in combination with hematopoietic stem cell transplantation. Thus, one possible explanation for the phenotype with respect to the overall survival (no association with the expression of B7-H6) could be the hypothesis that the use of response adjusted therapy might have affected the prognosis of T-LBL subjects. Moreover, another possibility could be the balance of the immunological and non-immunological roles of B7-H6 in patients with T-LBL (13). Although previous reports have shown the negative effect of the expression of $\mathrm{B} 7-\mathrm{H} 6$ on the survival of cancer patients, B7-H6 is an activating ligand of NKp30, a stimulating receptor on NK cells. Of note, studies have revealed that NKp30-positive NK/T cells are biomarkers for the favorable prognosis of melanoma $(27,28)$. Therefore, the potential negative effects of B7-H6 on patient survival are balanced by the positive role of B7H6 in the activation of NKp30-positive NK or NKT cells. Therefore, extensive lab testing and further studies focusing on the regimens used to treat T-LBL patients will help to better understand the pathobiology of T-LBL.

Our results suggest that B7-H6 may serve as a therapeutic target for the treatment of T-LBL. Although CD7 or CD5specific chimeric antigen receptor $\mathrm{T}$ cells (CAR-T) have shown preliminary success in early-stage clinical trials, this therapy is associated with several limitations and side effects (43-46). In this study, we observed membrane and cytoplasmic expression of B7-H6 in 38.5\% of the samples with weak to moderate staining intensity. B7-H6-specific therapies using NKp30 or antibodies have shown optimal responses in vitro (29-31). Similarly, we used NKp30based CAR-T cells, specifically targeting B7-H6 expressed on Jurkat cells, and observed in vitro tumor elimination (data not shown). Of note, B7-H6 was not detected on the membrane of other cells except activated monocytes, thereby highlighting its specificity. Importantly, patients with negative membrane expression of B7-H6, but with the presence of $\mathrm{B} 7-\mathrm{H} 6$ in the nucleus, benefit from the treatment with histone deacetylase inhibitors to reduce the expression of B7-H6 on activated monocytes and leukemia cells (34).

Notably, IHC suggested that B 7-H6 was a nucleocytoplasmic shuttling protein. Most of the previous reports have shown the expression of $\mathrm{B} 7-\mathrm{H} 6$ on the membrane or in the cytoplasm of several tumor cells 
$(15-24,26,35)$. Since the nuclear translocation of proteins is critical in tumorigenesis, we used the BioGRID system to predict any nuclear localization domains in $\mathrm{B} 7-\mathrm{H} 6$. B7-H6 was predicted to interact with XPO-5 and XPO6 , members of the karyopherin- $\beta$ family responsible for the nucleocytoplasmic shuttling of proteins. Of note, the binding domain of karyopherin- $\beta$ is well described and quite conserved (40).

The immunological effects of B7-H6 have been wellstudied. The non-immunological role of B7-H6 in signaling has also been reported (13). B7-H6 binds to NKp30 and activates NK cell toxicity. B7-H6 is associated with JAKSTAT signaling in lymphoma and several other solid cancers, improving tumor cell viability (21). However, this is the first report to show the nuclear localization of B7H6 in tumor cells; these results are in line with the role and properties of B7-H4 (47). Mutations in the NLS of B7$\mathrm{H} 4$ increased its surface expression and inhibited $\mathrm{T}$ cell proliferation and cytokine production. In vivo studies have shown that a mutant form of B7-H4 also impairs the G1/S phase transition in HEK293 cells, thereby reducing tumor cell proliferation and tumorigenicity (47). Importantly, wildtype $\mathrm{B} 7-\mathrm{H} 4$ is associated with chemosensitivity in rectal/colon cancer cell lines. However, in this study, we failed to determine a correlation between the nuclear localization of B7-H6 and patients' survival or prognosis (data not shown). This could be attributed to the limited sample size and the heterogeneity of treatments used in our cohort. Nevertheless, the findings in this study helped to unveil the effect of $\mathrm{B} 7-\mathrm{H} 6$ on the tumorigenesis of certain types of cancer. Although this study investigated the preliminary role of the NLS of B7-H6, future studies will help to provide mechanistic insight into the role of NLS in cancer progression.

We have previously shown that depleting NHL cells of B7-H6 inhibits tumor progression and enhances tumor chemosensitivity (21); moreover, increased apoptosis of B cell NHL was reported upon the depletion of B7-H6. In fact, we observed a correlation between the expression of B7-H6 and the response to chemotherapy in patients with T-LBL. It should be noted that chemotherapy may increase the mRNA and protein levels of B7-H6 and enhance the cytotoxicity of NK cells against cancer cells expressing B7-H6 (33). However, whether the chemotherapy- or radiation-induced overexpression of $\mathrm{B} 7-\mathrm{H} 6$ impairs sensitivity to chemotherapy remains unknown. Although enhanced recognition and toxicity by NK cells has been verified in vitro, the effects may not be reflected in vivo (33).
This said, several studies have shown the hydrolysis of B7H6 in the cytoplasm and subsequent downregulation of the NKp30 receptor on NK cells as a strategy for immune escape in various types of cancers (11-13,15,23,24).

Moreover, in this study, the results of RNAseq revealed that RAG-1 was differentially expressed in cells depleted of B7-H6. Interestingly, patients with T-ALL and B-ALL showed a high expression of RAG-1, determined as an important factor for cancer development (48-50). Unlike in the previous reports, here we failed to identify other differentially expressing genes related to the aggressiveness of T-LBL. In fact, since we obtained a limited set of differentially expressed genes after the depletion of B7H6, we conclude that B7-H6 is not likely a driver of tumorigenesis in T-LBL.

This study is not without limitations. First, due to the retrospective nature of the study, the clinical data obtained with the relatively small population failed to identify a correlation between the expression of B7-H6 and reduced survival in patients, and made a multivariate analysis impossible. Second, due to the clinical context, we could not use the $\mathrm{H}$ score to evaluate the expression of $\mathrm{B} 7-\mathrm{H} 6$ via IHC, unlike other studies $(14,15,17,18)$. However, we believe that our approach may be easier to perform and more helpful for daily clinical practice. Finally, owing to the scarcity of sufficient tissue mass (many tissues were collected by fine-needle aspiration), we failed to define the pathological diagnosis according to the origin of T-LBL.

\section{Conclusions}

Taken together, B7-H6 may be a negative prognostic factor and potential therapeutic target of T-LBL. B7-H6 levels in Jurkat cells correlated with the functioning of multiple signaling pathways. Proliferation, migration, and invasion were impaired by the downregulation of B7-H6. Moreover, nuclear translocation of B7-H6 may play an important role in the pathogenesis of T-LBL.

\section{Acknowledgments}

The authors thank Dr. Jinlei Qi from National Center for Chronic and Noncommunicable Disease Control and Prevention, Chinese Center for Disease Control and Prevention for the statistical assistance and Dr. Xiaolong Liu, Professor Zifen Gao from Department of Pathology, Peking University Health Science Center for the technical assistance. We thank Dr. Fei Dong and Dr. Qihui Li for the 
assistance in collecting clinical information. The authors also thank Editage.com for the language editing assistance. Funding: This work was supported by National Natural Science Foundation of China (Grant No. 81670181 to XK). Innovation Fund for Outstanding Doctoral Candidates of Peking University Health Science Center (Grant No. 71013 Y2029 to LY).

\section{Footnote}

Reporting Checklist: The authors have completed the REMARK reporting checklist. Available at http://dx.doi. org/10.21037/atm-20-5308

Data Sharing Statement: Available at http://dx.doi. org/10.21037/atm-20-5308

Conflicts of Interest: All authors have completed the ICMJE uniform disclosure form (available at http://dx.doi. org/10.21037/atm-20-5308). The authors have no conflicts of interest to declare.

Ethical Statement: The authors are accountable for all aspects of the work in ensuring that questions related to the accuracy or integrity of any part of the work are appropriately investigated and resolved. The study was conducted in accordance with the Declaration of Helsinki (as revised in 2013). The study was approved by the Institutional Review Board of Peking University Third Hospital (Number: 2016060). Due to the study's retrospective nature, written informed consent was waived.

Open Access Statement: This is an Open Access article distributed in accordance with the Creative Commons Attribution-NonCommercial-NoDerivs 4.0 International License (CC BY-NC-ND 4.0), which permits the noncommercial replication and distribution of the article with the strict proviso that no changes or edits are made and the original work is properly cited (including links to both the formal publication through the relevant DOI and the license). See: https://creativecommons.org/licenses/by-nc-nd/4.0/.

\section{References}

1. Han X, Kilfoy B, Zheng T, et al. Lymphoma survival patterns by WHO subtype in the United States, 19732003. Cancer Causes Control 2008;19:841-58.

2. Boucheix C, David B, Sebban C, et al. Immunophenotype of adult acute lymphoblastic leukemia, clinical parameters, and outcome: an analysis of a prospective trial including 562 tested patients (LALA87). French Group on Therapy for Adult Acute Lymphoblastic Leukemia. Blood 1994;84:1603-12.

3. Dores GM, Devesa SS, Curtis RE, et al. Acute leukemia incidence and patient survival among children and adults in the United States, 2001-2007. Blood 2012;119:34-43.

4. Chiaretti S, Vitale A, Cazzaniga G, et al. Clinico-biological features of 5202 patients with acute lymphoblastic leukemia enrolled in the Italian AIEOP and GIMEMA protocols and stratified in age cohorts. Haematologica 2013;98:1702-10.

5. Marks DI, Paietta EM, Moorman AV, et al. T-cell acute lymphoblastic leukemia in adults: clinical features, immunophenotype, cytogenetics, and outcome from the large randomized prospective trial (UKALL XII/ECOG 2993). Blood 2009;114:5136-45.

6. Harrison CJ. Cytogenetics of paediatric and adolescent acute lymphoblastic leukaemia. Br J Haematol 2009;144:147-56.

7. Gianni F, Belver L, Ferrando A. The Genetics and Mechanisms of T-Cell Acute Lymphoblastic Leukemia. Cold Spring Harb Perspect Med 2020;10:a035246.

8. Belver L, Ferrando A. The genetics and mechanisms of T cell acute lymphoblastic leukaemia. Nat Rev Cancer 2016;16:494-507.

9. Knoechel B, Bhatt A, Pan L, et al. Complete hematologic response of early T-cell progenitor acute lymphoblastic leukemia to the -secretase inhibitor BMS-906024: genetic and epigenetic findings in an outlier case. Cold Spring Harb Mol Case Stud 2015;1:a000539.

10. Papayannidis C, DeAngelo DJ, Stock W, et al. A Phase 1 study of the novel gamma-secretase inhibitor PF03084014 in patients with T-cell acute lymphoblastic leukemia and T-cell lymphoblastic lymphoma. Blood Cancer J 2015;5:e350.

11. Baratin M, Vivier E. B7-H6: a novel alert signal for NK cells. Med Sci (Paris) 2010;26:119-20.

12. Brandt CS, Baratin M, Yi EC, et al. The B7 family member $\mathrm{B} 7-\mathrm{H} 6$ is a tumor cell ligand for the activating natural killer cell receptor NKp30 in humans. J Exp Med 2009;206:1495-503.

13. Hu Y, Zeng T, Xiao Z, et al. Immunological role and underlying mechanisms of $\mathrm{B} 7-\mathrm{H} 6$ in tumorigenesis. Clin Chim Acta 2020;502:191-8.

14. Chen H, Guo Y, Sun J, et al. Preferential Expression of B7-H6 in Glioma Stem-Like Cells Enhances Tumor Cell 
Proliferation via the c-Myc/RNMT Axis. J Immunol Res 2020;2020:2328675.

15. Gutierrez-Silerio GY, Franco-Topete RA, Haramati $\mathrm{J}$, et al. Positive staining of the immunoligand B7-H6 in abnormal/transformed keratinocytes consistently accompanies the progression of cervical cancer. BMC Immunol 2020;21:9.

16. Cao Y, Huo L, Zhou L, et al. Expression of B7-H6 in chronic myeloid leukemia and its clinical significance. Int J Clin Exp Pathol 2019;12:568-75.

17. Zhou H, Dong J, Guo L, et al. The prognostic value of B7-H6 in esophageal squamous cell carcinoma. Sci Rep 2019;9:18122.

18. Chen L, Feng J, Xu B, et al. Correction to: Expression of B7-H6 expression in human hepatocellular carcinoma and its clinical significance. Cancer Cell Int 2018;18:134.

19. Chen L, Feng J, Xu B, et al. B7-H6 expression in human hepatocellular carcinoma and its clinical significance [corrected]. Cancer Cell Int 2018;18:126.

20. Guo JG, Guo CC, He ZQ, et al. Clinical significance of B7-H6 protein expression in astrocytoma. Onco Targets Ther 2016;9:3291-7.

21. Wu F, Wang J, Ke X. Knockdown of B7-H6 inhibits tumor progression and enhances chemosensitivity in B-cell non-Hodgkin lymphoma. Int J Oncol 2016;48:1561-70.

22. Zhou Y, Xu Y, Chen L, et al. B7-H6 expression correlates with cancer progression and patient's survival in human ovarian cancer. Int J Clin Exp Pathol 2015;8:9428-33.

23. Pesce S, Tabellini G, Cantoni C, et al. B7-H6-mediated downregulation of NKp30 in NK cells contributes to ovarian carcinoma immune escape. Oncoimmunology 2015;4:e1001224.

24. Semeraro M, Rusakiewicz S, Minard-Colin V, et al. Clinical impact of the NKp30/B7-H6 axis in high-risk neuroblastoma patients. Sci Transl Med 2015;7:283ra55.

25. Zhang X, Xie W, Wang Z, et al. Expression of a novel immune checkpoint B7-H6 ligand in human small cell lung cancer. Ann Transl Med 2020;8:589.

26. Chen XJ, Shen J, Zhang GB, et al. B7-H6 protein expression has no prognostic significance in human gastric carcinoma. Pathol Oncol Res 2014;20:203-7.

27. Messaoudene M, Fregni G, Enot D, et al. NKp30 isoforms and NKp46 transcripts in metastatic melanoma patients: Unique NKp30 pattern in rare melanoma patients with favorable evolution. Oncoimmunology 2016;5:e1154251.

28. Correia MP, Stojanovic A, Bauer K, et al. Distinct human circulating NKp30+Fc RI +CD8+ T cell population exhibiting high natural killer-like antitumor potential.
Proc Natl Acad Sci U S A 2018;115:E5980-9.

29. Zhang T, Wu MR, Sentman CL. An NKp30-based chimeric antigen receptor promotes $\mathrm{T}$ cell effector functions and antitumor efficacy in vivo. J Immunol 2012;189:2290-9.

30. Gacerez AT, Hua CK, Ackerman ME, et al. Chimeric antigen receptors with human scFvs preferentially induce $\mathrm{T}$ cell anti-tumor activity against tumors with high B7H6 expression. Cancer Immunol Immunother 2018;67:749-59.

31. Wu MR, Zhang T, Gacerez AT, et al. B7H6Specific Bispecific T Cell Engagers Lead to Tumor Elimination and Host Antitumor Immunity. J Immunol 2015;194:5305-11.

32. Schlecker E, Fiegler N, Arnold A, et al. Metalloproteasemediated tumor cell shedding of $\mathrm{B} 7-\mathrm{H} 6$, the ligand of the natural killer cell-activating receptor NKp30. Cancer Res 2014;74:3429-40.

33. Cao G, Wang J, Zheng X, et al. Tumor Therapeutics Work as Stress Inducers to Enhance Tumor Sensitivity to Natural Killer (NK) Cell Cytolysis by Up-regulating NKp30 Ligand B7-H6. J Biol Chem 2015;290:29964-73.

34. Fiegler N, Textor S, Arnold A, et al. Downregulation of the activating NKp30 ligand $\mathrm{B} 7-\mathrm{H} 6$ by HDAC inhibitors impairs tumor cell recognition by NK cells. Blood 2013;122:684-93.

35. Zhang X, Zhang G, Qin Y, et al. B7-H6 expression in non-small cell lung cancers. Int J Clin Exp Pathol 2014;7:6936-42.

36. Gillis S, Watson J. Biochemical and biological characterization of lymphocyte regulatory molecules. V. Identification of an interleukin 2-producing human leukemia T cell line. J Exp Med 1980;152:1709-19.

37. Oughtred R, Stark C, Breitkreutz BJ, et al. The BioGRID interaction database: 2019 update. Nucleic Acids Res 2019;47:D529-41.

38. Stark C, Breitkreutz BJ, Reguly T, et al. BioGRID: a general repository for interaction datasets. Nucleic Acids Res 2006;34:D535-9.

39. Bennasser Y, Chable-Bessia C, Triboulet R, et al. Competition for XPO5 binding between Dicer mRNA, pre-miRNA and viral RNA regulates human Dicer levels. Nat Struct Mol Biol 2011;18:323-7.

40. Lee BJ, Cansizoglu AE, Süel KE, et al. Rules for nuclear localization sequence recognition by karyopherin beta 2 . Cell 2006;126:543-58.

41. Marín-Rubio JL, Pérez-Gómez E, Fernández-Piqueras J, et al. S194-P-FADD as a marker of aggressiveness and 
poor prognosis in human T-cell lymphoblastic lymphoma. Carcinogenesis 2019;40:1260-8.

42. Kelwick R, Desanlis I, Wheeler GN, et al. The ADAMTS (A Disintegrin and Metalloproteinase with Thrombospondin motifs) family. Genome Biol 2015;16:113

43. Xu Y, Liu Q, Zhong M, et al. 2B4 costimulatory domain enhancing cytotoxic ability of anti-CD5 chimeric antigen receptor engineered natural killer cells against $\mathrm{T}$ cell malignancies. J Hematol Oncol 2019;12:49.

44. Raikar SS, Fleischer LC, Moot R, et al. Development of chimeric antigen receptors targeting $\mathrm{T}$-cell malignancies using two structurally different anti-CD5 antigen binding domains in NK and CRISPR-edited T cell lines. Oncoimmunology 2017;7:e1407898.

45. Png YT, Vinanica N, Kamiya T, et al. Blockade of CD7 expression in $\mathrm{T}$ cells for effective chimeric antigen receptor targeting of T-cell malignancies. Blood Adv 2017;1:2348-60.

Cite this article as: Yuan L, Sun L, Yang S, Chen X, Wang J, Jing H, Zhao Y, Ke X. B7-H6 is a new potential biomarker and therapeutic target of T-lymphoblastic lymphoma. Ann Transl Med 2021;9(4):328. doi: 10.21037/atm-20-5308
46. Chen KH, Wada M, Pinz KG, et al. Preclinical targeting of aggressive T-cell malignancies using anti-CD5 chimeric antigen receptor. Leukemia 2017;31:2151-60.

47. Zhang L, Wu H, Lu D, et al. The costimulatory molecule B7-H4 promote tumor progression and cell proliferation through translocating into nucleus. Oncogene 2013;32:5347-58.

48. Gladdy RA, Taylor MD, Williams CJ, et al. The RAG-1/2 endonuclease causes genomic instability and controls CNS complications of lymphoblastic leukemia in p53/Prkdcdeficient mice. Cancer Cell 2003;3:37-50.

49. Kawakubo K, Ohyashiki JH, Ohyashiki K, et al. Hypomethylated status, but not RAG-1, is required for T-cell receptor-beta-chain gene rearrangement in acute leukemia cells. Cancer Genet Cytogenet 1994;78:40-5.

50. Umiel T, Pattengale P, Weinberg K. Recombination activating gene-1 (RAG-1) expression in all differentiation stages of B-lineage precursor acute lymphoblastic leukemia. Leukemia 1993;7:435-40. 